Case Report

Volume 1 - Issue 1

\title{
Ascending Posterior Mediastinal Abscess from Vertebral Osteomyelitis - A Case Report and Review of Diagnosis and Management
}

Miriam Oren ${ }^{1}$, Daniella Ohnemus ${ }^{1}$, Ezeldeen Abuelkasem ${ }^{1}$ and Michael S Trostler ${ }^{1, *}$

${ }^{1}$ University of Pittsburgh Medical Center, Pittsburgh, PA, USA

*Corresponding author: Michael Trostler, Anesthesiology and Perioperative Medicine, University of Pittsburgh Medical Center, Pittsburgh, PA, USA

Received date: 4 June, 2021 | Accepted date: 10 July, 2021 | Published date: 13 July, 2021

Citation: Oren M, Ohnemus D, Abuelkasem E, Trostler MS (2021) Ascending Posterior Mediastinal Abscess from Vertebral Osteomyelitis - A Case report and Review of Diagnosis and Management. J Anaesth Anesth Drug 1(1). doi https://doi.org/10.54289/JAAD2100101

Copyright: () 2021 Oren M, et al. This is an open-access article distributed under the terms of the Creative Commons Attribution License, which permits unrestricted use, distribution, and reproduction in any medium, provided the original author and source are credited.

Abbreviations: VATS: Video Assisted Thoracoscopic Surgery, MSSA: Methicillin-Susceptible Staphylococcus Aureus, MRI: Magnetic Resonance Imaging.

\section{Introduction}

Vertebral osteomyelitis is characterized by inflammation and infection of the vertebrae that primarily arises from hematogenous spread or via a direct route. The presenting symptom is most commonly back pain, which if left untreated, can extend to adjacent tissues or organs such as the spinal column, epidural space and pleural space, and lead to life-threatening complications such as mediastinitis. We present here the case of a 70-year-old woman with a mediastinal abscess in the setting of vertebral osteomyelitis.

\section{Case:}

MS, a 70-year-old female, presents to the ER with 3-4 days of worsening back pain, and was incidentally found to be COVID positive but was not displaying any specific symptoms. She has a history of posterior spinal fusion L2-S1 which was complicated by methicillin-susceptible Staphylococcus aureus (MSSA) bacteremia, osteomyelitis and discitis requiring removal of hardware and prolonged antibiotic course which was completed 6 months prior. She now presents with a T12 compression fracture with osteomyelitis, a mediastinal abscess, and MSSA bacteremia.

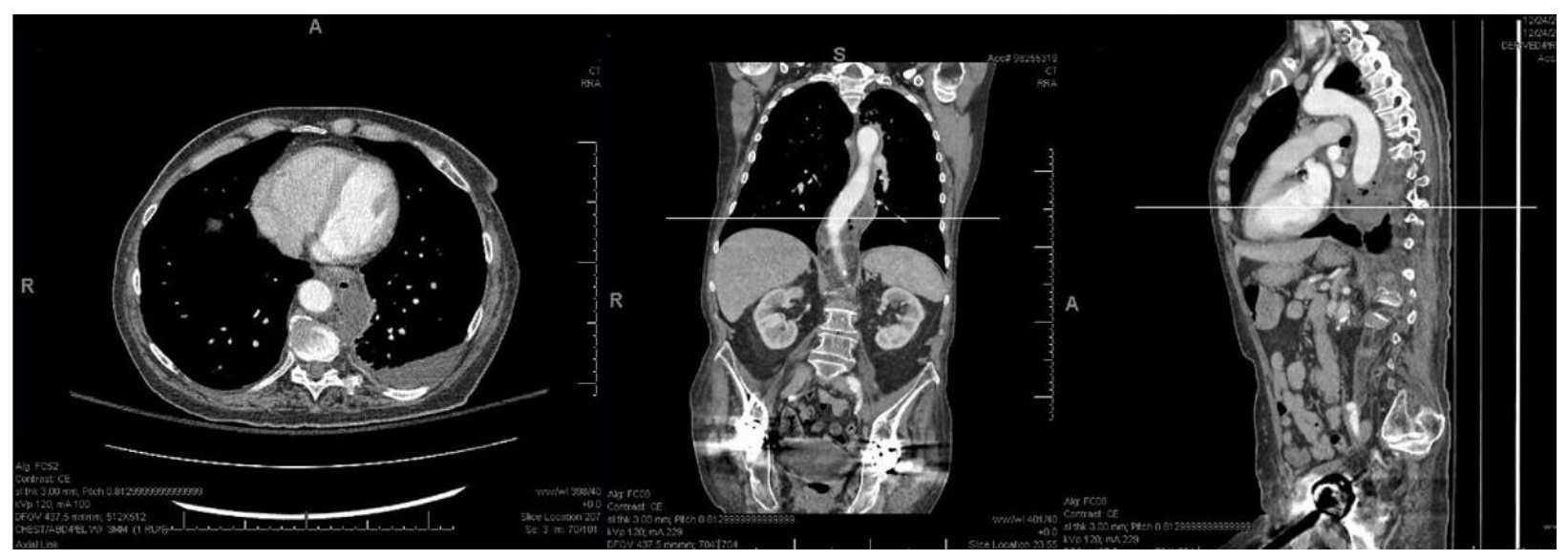

Figure 1. CT Chest demonstrating the posterior mediastinal abscess in axial(left), coronal(middle), and sagittal(right) planes. 
The patient presented to the OR for left video assisted thoracoscopic surgery (VATS) drainage of a periaortic, posterior mediastinal abscess as seen in the CT scan in figure 1. A preinduction radial arterial line was placed and the patient was intubated via rapid sequence intubation with a left $37 \mathrm{Fr}$ double lumen endotracheal tube. The patient was carefully placed in right lateral decubitus position on a beanbag with less flexion than is normally applied in this position as there was concern that her spine fractures may not be fully stable. The patient was prepped in the usual sterile manner and the left lung isolated. Upon entry to the chest cloudy amber fluid was noted in the posterior gutter. A posterior mediastinal abscess was found encompassing the aorta from the start of the descending aorta to level of the diaphragm. The abscess was unroofed and frank purulence was washed out with copious antibiotic irrigation. The patient was returned to the supine position and the double lumen tube was exchanged for a single lumen. Bronchoscopy revealed moderate secretions and a bronchoalveolar lavage was performed. Upper endoscopy found no signs of injury that might have caused this abscess. The patient was left intubated postoperatively to allow the lung time to re-expand and for any translocation of bacteria to clear. The patient was extubated 24 hours later in the ICU.

Postoperative the patient underwent a thorough workup including a transthoracic echocardiogram which did not identify any vegetations, an MRI (Magnetic Resonance Imaging) Thoracic/Lumbar-spine with and without contrast demonstrated discitis/osteomyelitis of T11-T12 with extensive abscess formation within the T12 vertebral body and extensive epidural phlegmon throughout the thoracic and lumbar spine, and evidence of intrathecal spread of infection (Figure 1-B) Blood cultures, mediastinal and pleural cultures grew MSSA. She was discharged home 4 weeks after admission on a course of antibiotics with plan for at least 6 weeks of treatment from blood culture clearance in addition to repeat $\mathrm{CT}$ chest and MRI T/L-spine prior to stopping antibiotics.

\section{Discussion:}

This is a very rare case of ascending posterior mediastinal abscess or ascending peri-aortic abscess originating from vertebral osteomyelitis/discitis and epidural abscess. This specific etiology and presentation have only been described in the literature twice, first a case report from 1966 had found two infants that presented with mediastinal abscesses from vertebral osteomyelitis, one arising spontaneously at age 7weeks, the second in a 12-week-old infant arising from bacteremia from an exchange transfusion for erythrocytosis performed at age 2 weeks. Secondly in 1982 where a patient diagnosed with prostatitis and then presented with bilateral pleural effusions and an abscess extending from the thoracic apex to the aortic arch originating from the T2 vertebrae. [1,2] Vertebral osteomyelitis can be caused by hematogenous seeding from distant sites, contiguous spread from adjacent tissues, or invasive spinal procedures [3]. When a known focus of vertebral osteomyelitis is present it can spread into adjacent structures and along body planes, making it highly likely to be the primary nidus of infection in our case [4]. The diagnosis of vertebral osteomyelitis can be difficult to make; a combination of clinical findings, laboratory studies, and radiographic evidence is usually required.

Osteomyelitis can be challenging to detect with X-ray films, as they often appear normal initially, so the gold standard for detection is MRI with contrast [4]. As it can spread into adjacent structures, once the site is discovered it is important to assess the surrounding areas for evidence of infection. The most common symptom on presentation is back pain, but patients may present with more clinically apparent symptoms such as shortness of breath caused by a pleural effusion [3]. A CT scan of the neck and chest is an important diagnostic test to assess for mediastinal and pleural involvement, and a pleural effusion in a patient also reporting back pain should raise suspicion for vertebral osteomyelitis [5]. Treatment of any abscess involves source control and debridement in addition to organism specific antibiotics [6].

The vertebral, epidural, and intrathecal spaces are anatomically close to the pleural space, making contiguous spread a real concern in either direction, with approximately one third of epidural abscesses arising this way [7,8]. Staphylococcus aureus is the most common causative organism, causing about two thirds of cases due to invasion of skin flora, as we saw in our case [8]. We additionally had 
spread of bacteria to the blood, mediastinum, and pleural spaces, but more commonly these spaces will seed the vertebrae which is common in endocarditis. Thoracolumbar abscesses are more common than other areas of the spine as the space is larger and there is more infection-prone fat [8]. Mediastinitis is an uncommon disease process. Other causes include previous cardiac surgery, descending necrotizing mediastinitis from the retropharyngeal space, esophageal perforation, esophageal fistula, tuberculosis, or mycotic infections [6,9]. Therefore, it is important to perform an oral exam and endoscopy in cases of mediastinitis. However, if the cause for the mediastinitis has not been established, osteomyelitis should be kept on the differential diagnosis.

In conclusion, osteomyelitis is an insidious disease that is often found late due to a variable presentation and can have severe neurologic consequences if left untreated. When intrathoracic infection of unknown etiology is present, it is important to keep vertebral osteomyelitis on the differential as it can be a consequence of distant infection or the primary source.

Conflict of Interest: Authors declare no conflict of interest

Funding: None

\section{References:}

1. Benezra C, Spurgeon L, Light RW (1982) Mediastinal abscess secondary to vertebral osteomyelitis. Postgrad Med 71 (3): 220-223.
2. DeLorimer AA (1966) Mediastinal Mass Caused by Vertebral Osteomyelitis. Am J Dis Child 111 (6): 639643.

3. Sohatee MA, Shields DW (2013) Painless vertebral osteomyelitis: an unusual presentation. BMJ Case Rep 2013: bcr2012008298.

4. Bass SN, Ailani RK, Shekar R, Gerblich AA (1998) Pyogenic Vertebral Osteomyelitis Presenting as Exudative Pleural Effusion. Chest 114 (2): 642-647.

5. Dzian A, Stiegler P, Smolár M, Hamzik J, Mistuna D (2013) Posterior mediastinotomy as an unordinary method of mediastinal drainage in patient with descending necrotizing mediastinitis: a case report. Thorac Cardiovasc Surg 61(2): 175-177.

6. Mandell GL, Bennett JE, Dolin R (2009) Mandell G, Bennett J, Dolin R, eds. Principles and Practice of Infectious diseases. Seventh edition. Oxford Elsevier 1173-1182.

7. Nakashima T, Sagishima K, Suenaga H, Yamamoto T (2020) Untreated Vertebral Osteomyelitis Extending to the Mediastinum and Lungs. BMJ Case Reports 13(8): e236249.

8. Darouiche RO (2006) Spinal epidural abscess. N Engl J Med 355(9): 2012-2020.

9. Brook I, Frazier EH (1996) Microbiology of mediastinitis. Arch Intern Med 156(3): 333-336. 